\title{
Três Minas - Eine Bergwerkskatastrophe mit Rettungsaktion in der römischen Kaiserzeit
}

\author{
Regula Wahl-Clerici, Markus Helfert, Annemarie Wiechowski
}

\begin{abstract}
Zusammenfassung
Im römischen Goldbergwerksdistrikt von Três Minas (Freg. Três Minas, Conc. Vila Pouca de Aguiar / Portugal) haben sich in einem seitlichen Treppenschacht der Galeria dos Alargamentos Spuren eines Grubenunglücks und einer darauf folgenden Rettungsaktion erhalten. Dieser bisher einmalige Befund stellt eine interessante Bereicherung für die antike Bergbauforschung dar.
\end{abstract}

In den Abbauzonen von Três Minas, Gralheira und Campo de Jales (Abb. 1) wurden im 1. und 2. Jh. n. Chr. polymetallische Erze mit hohen Gold- und Silbergehalten unter kaiserlicher Regie abgebaut ${ }^{1}$. Die römischen Überreste in Campo de Jales wurden durch den erneuten Abbau im Verlauf des 20. Jh. weitgehend zerstört. Der Erzgang von Gralheira blieb bislang abgesehen von verschiedenen Prospektionsaktivitäten von mehr oder weniger einschneidenden Maßnahmen verschont ${ }^{2}$. Am aufwändigsten war der Abbau in der Vererzungszone von Três Minas (Abb. 2), wo der Erhaltungszustand der Monumente dank des standfesten Gesteins und der eher geringen Prospektionsmaßnahmen als außerordentlich gut zu bezeichnen ist. Erst hierdurch ist es möglich, die Spuren des Bergbaus in ihrem originalen Kontext zu erforschen und zu verstehen ${ }^{3}$.

In der Abbauzone von Três Minas dominieren heute die beiden mächtigen Tagebaue Corta de Covas und Corta da Ribeirinha, deren Längsachsen in einer Linie parallel zum Streichen des Gesteins verlaufen. Die beiden Tagebaue wurden mehrfach durch querschlägig, d.h. quer zum Streichen des anstehenden Schiefers, angelegte Untertagebaue in den tieferen Lagen erschlossen. Diese Baue, meist handelt es sich streng genommen um Tunnels, dienten der Entwässerung der Tagebaue. Gleichzeitig wurden sie zum Transport des abgebauten Materials genutzt. Die lichte Weite der Stollen und die in der Sohle erkennbaren Spurrinnen belegen, dass rollende Förderung mittels von Rindern gezogenen Karren betrieben wurde ${ }^{4}$.

Die Galeria dos Alargamentos folgt als einziger Untertagebau dem Streichen der Gesteine. Der großzügige Ausbau, die erhaltenen Karrenspuren sowie die vermeintliche Verbindung zur Corta de Covas ließen bis in jüngste Zeit vermuten, dass dieser Bau dem gleichen Zweck diente wie die querschlägigen Galerien ${ }^{5}$. Erst durch die Forschungen der letzten Jahre wurde ihre eigentliche Funktion im Rahmen der römischen Prospektion erkannt ${ }^{6}$. Wesentlichen Einfluss auf diese Neuinterpretation hatte die Vermessung der Galeria dos Alargamentos mittels 3D-Laserscanning, die im

\footnotetext{
${ }^{1}$ WAHL 1988, 221-244.

${ }^{2}$ Dr. Domingos Dias, der Präsident der Câmara Municipal von Vila Pouca de Aguiar, gab im Oktober 2011 die verbindliche Auskunft, dass die Wiederaufnahme der Ausbeutung der Erzlagerstätte von Gralheira für 2012 geplant ist.

${ }^{3}$ WAHL-CLERICI/WIECHOWSKI, IM DRUCK; WAHL-CLERICI, IM DRUCK.

${ }^{4}$ WAHL-CLERICI 2008, 56; WAHL-CLERICI 2010, Abb. 6.

${ }^{5}$ WAHL 1988, $229 \mathrm{f}$.

${ }^{6}$ WAHL-CLERICI/HELFERT/RAMMINGER 2011; WAHL-CLERICI/WIECHOWSKI, IM DRUCK.
} 
Rahmen des Projekts zur Erforschung des Bergwerksdistrikts von Três Minas mit nicht-invasiven Methoden durchgeführt wurde ${ }^{7}$. Tatsächlich bildet die Galeria dos Alargamentos zusammen mit zwei Schächten in der Ostwand der Corta de Covas sowie weiteren Schächten und Stollen ein ganzes System zur Beprobung der zwischen den beiden Tagebauen liegenden Zone (Abb. 3-5). Zu diesem Prospektionssystem gehört auch ein im oberen Teil der Hangoberfläche aufgefahrener tonnlägiger (schräger) Schacht sowie eine kleine Probenentnahmestelle im Bereich des Mundlochs der Galerie. Im Berginnern, in einer großen Kaverne am Ende der Galeria dos Alargamentos, treffen Stollen und Förderschacht aufeinander, wobei der Schacht die Sohle der Kaverne durchstößt (Abb. 6). Diese 4,15 m hohe und max. 3,50 m weite Verbindung bot Platz für einen Göpel, der zur weiteren Abteufung des Förderschachts notwendig war. Zudem wurden weitere Prospektionsstollen in Richtung Westen aufgefahren, die meisten wohl erst nach Auflassung der Schachtabteufung und des dazu nötigen Hebewerks mit Göpelantrieb. Dies trifft auch auf einen in der Mitte der Göpelrundung abgeteuften Schacht zu.

$\mathrm{Zu}$ diesen Prospektionsbauten, die erst nach der Einstellung des Schachtbetriebs angelegt wurden, gehört auch der in den südlichen Stoß aufgefahrene Treppenschacht (Abb. 7). Die in die Sohle der Galerie eingeschlagenen obersten Treppenstufen hätten sonst die Durchfahrt mit Karren zu sehr behindert. Der Schacht mit heute noch insgesamt 14 sichtbaren Stufen ist nicht ganz gerade, sondern beschreibt eine leichte Kurve nach Osten. Die lichte Weite beträgt $1,15 \mathrm{~m}$, die lichte Höhe variiert zwischen 1,60 m (Stufe 2), 1,90 m (ausgebrochene Stelle in der Firste bei Stufe 3) und 1,55 m (Stufe 13). Mit diesen Stufen wurden eine Distanz von rund $5 \mathrm{~m}$ und eine Höhe von $2,75 \mathrm{~m}$ überwunden.

Nach Stufe 14, die nur noch im östlichen Teil erhalten ist, bricht die Treppe an einem ca. 40 bis $50 \mathrm{~cm}$ breiten Spalt ab (Abb. 8 u. 9). Hier ist ein ca. $5 \mathrm{~m}$ langer Felsblock (Abb. 8 u. 9, Block 1) samt dem eingearbeiteten Treppenschacht nach unten versetzt worden. Dies geschah entlang von Klüften, die etwa senkrecht zueinander verlaufen. Es handelt sich erstens um Klüfte, die der Schichtung und Schieferung der Gesteine (ca. NNW-OSO, N $120^{\circ}$ ) entsprechen und steil nach NNO einfallen $\left(75^{\circ}\right.$ ), sowie zweitens um etwa NNW-SSO (N 20 $)$ streichende, ebenfalls steil einfallende $\left(75^{\circ}\right)$ Klüfte, die etwa parallel zum Schacht verlaufen. Bei letzteren handelt es sich um Querklüfte, die überall im Gebiet anzutreffen sind (z. B. Ostwand des Tagebaus A, Abb. 4). Steigt man die 14 Stufen des Schachtes hinab, blickt man auf eine Felswand (Abb. 12), welche die Verwerfungsfläche darstellt, an der die Treppe um 22,20 m nach unten versetzt wurde. Der hier entstandene, oben erwähnte Spalt ist mit Gesteinsbruchstücken verfüllt. Ein über der Treppe in dem Spalt natürlich verkeilter Felsbrocken verhindert das Nachrutschen von größerem Gesteinsmaterial. Der nach unten versetzte Schacht setzt sich zunächst weiter fort (Abb. 8 u. 9). Zwar ist die Sohle des Schachts wegen des Schuttes nicht sichtbar, aber an der Firste sowie am westlichen Stoß sind die Spuren der römischen Ausarbeitung deutlich zu erkennen, während der östliche Stoß stark ausgebrochen ist und sich zum nächsten Spalt weitet (Abb. 10). Ungefähr $5 \mathrm{~m}$ weiter in Richtung des Treppenschachts kam es an einem weiteren, ungefähr $30 \mathrm{~cm}$ breiten Spalt zum erneuten Abbruch des Schachtes in die Tiefe, diesmal um 1,4 m (Abb. 8. 9). Dabei wurde der Felsblock (Abb. 8. 9, Block 2) mit dem Treppenschacht nicht nur nach unten verworfen, sondern auch um $40 \mathrm{~cm}$ in östliche

\footnotetext{
${ }^{7}$ Organisatorische Leitung: Jun. Prof. Dr. B. Ramminger (Universität Hamburg); Forschungsleitung: Dr. Markus Helfert (Universität Hamburg) und lic-phil. Regula Wahl-Clerici (Universität Hamburg).
} 
Richtung versetzt. Die Firste des abgesenkten Schachts ist in der Felswand zu sehen. $\mathrm{Ab}$ hier ist er vollständig mit Schutt verfüllt.

Der Treppenschacht wurde nach dem Versturz bzw. den Verstürzen wiederum begangen. Dafür sprechen drei Lampennischen, welche in die von der Galeria dos Alargamentos aus sichtbare Felswand beim ersten Spalt eingearbeitet wurden. Diese Nischen können nur nach dem Bergereignis (Bergsturz) angebracht worden sein, da der Block erst durch das Absenken der Zone überhaupt freigelegt wurde. Bei der tiefer liegenden Abbruchstelle D wurden ebenfalls Nischen in die nach dem Ereignis freiliegende Felswand eingearbeitet (Abb. 8-10). Dabei handelt es sich um zwei runde Vertiefungen mit je einem Durchmesser von $11 \mathrm{~cm}$, die in einem Abstand von $14 \mathrm{~cm}$ angeordnet sind. Ihre Funktion konnte bislang nicht bestimmt werden. Ungefähr in der Mitte über den beiden Vertiefungen wurde $20 \mathrm{~cm}$ höher eine Lampennische in den Fels gehauen. Des Weiteren wurde eine Trockenmauer bei B-C, die offenbar vor weiterem Versturzmaterial aus dem Spalt sichern sollte, auch erst nach dem Absinken des Treppenschachts gebaut (Abb. 11).

Alle diese Befunde lassen sich nur erklären, wenn nach dem Bergsturz eine Rettungsaktion durchgeführt wurde, um Bergleute und Geräte, die sich zum Zeitpunkt der Katastrophe im Treppenschacht befanden, zu bergen. Als weiteres Argument für diese Interpretation spricht die Tatsache, dass der Treppenschacht in späterer Zeit nicht mehr zur weiteren Nutzung aufgewältigt, d. h. wiederhergestellt, wurde. So blieb der nicht erweiterte Durchgang von B nach $\mathrm{C}$ in seiner verstürzten Form unberührt (Abb. 12).

Ebenfalls für eine Rettungsaktion sprechen zwei bei Stufe 14, direkt unterhalb der Firste gegenüberliegend angebrachte Einarbeitungen, die dazu verhalfen einen Balken von max. $6 \mathrm{~cm}$ Durchmesser zu verkeilen. Im östlichen Stoß handelt es sich um eine Einarbeitung mit $6 \mathrm{~cm}$ Höhe und $9 \mathrm{~cm}$ Länge. Im westlichen Stoß wurde zusätzlich eine Nut zum Befestigen und Sichern des Balkens eingemeißelt. Wahrscheinlich diente dieser Balken zur Befestigung einer Seilwinde, die konstruiert wurde, um Schuttmaterial aus dem Treppenschacht schneller herauszuziehen. In der Galeria dos Alargamentos finden sich im Bereich des Treppenschachts mindestens drei Einarbeitungen, die tiefer als die übrigen als Lampennischen identifizierten Einarbeitungen liegen. Damit wurde möglicherweise die zur Sicherung der Seilwinde nötige Konstruktion abgestützt. Es ist anzunehmen, dass der untere Abschnitt des Treppenschachtes direkt nach dem Ereignis noch nicht so verfüllt war, wie er sich heute darstellt, und erst im Verlauf der Jahrhunderte zusätzlicher Abraum in den Schacht gelangte.

Die Frage nach der Ursache für das Bergereignis vermögen die nachweisbaren Befunde nicht zu beantworten. Als Auslöser für die Verwerfung des Treppenschachtes kommt zunächst ein Erdbeben als Folge von tektonischen Bewegungen in Frage. Weiterhin wäre ein Einsturz von Hohlräumen möglich, die mit dem Abbau im Tagebau oder dem Förderschacht im Zusammenhang standen und unterhalb der Zone des Treppenschachtes lagen (Abb. 3). In beiden Fällen wären Erderschütterungen wahrgenommen worden, und beide Ursachen würden das gleiche Ergebnis liefern ${ }^{8}$.

Um entscheiden zu können, welche Möglichkeit die wahrscheinlichere ist, müssten Recherchen über die seismischen Aktivitäten in der Gegend sowie Beobach-

\footnotetext{
${ }^{8}$ WALDHERR 1997, 75. - Mit der von Plinius beschriebenen Abbautechnik der arrugiae (n.h. 33, 70 ff.) wurden durch die systematische Unterhöhlung Teile eines Berges in sekundären Lagerstätten zum Einsturz gebracht. Es finden sich keine Hinweise für diese Technik in Três Minas im Gegensatz zu Las Médulas (Prov. León, Spanien). S. a. Domergue 2008, 138.
} 
tungen an der Erdoberfläche durchgeführt werden ${ }^{9}$. Die im interdisziplinären Workshop vom 14./15. Mai 2004 vorgestellten Beispiele zum Thema „Antike Erdbeben im alpinen und zirkumalpinen Raum" zeigen überaus deutlich auf, wie schwierig Erdbeben allein anhand archäologischer Befunde zu belegen sind ${ }^{10}$. Was auch immer der Auslöser war, wir sind Zeugen der Bemühungen der römischen Bergleute, ihre betroffenen Kumpels zu retten oder wenigstens zu bergen.

Die Katastrophenforschung hat sich in den letzten 20 Jahren ausführlich mit der Überlieferung und Wahrnehmung von Naturereignissen, vor allem Erdbeben, Seebeben mit Überflutungen (Tsunamis) und Vulkanausbrüchen in der Antike auseinander gesetzt. Es überwiegen größere Vorkommnisse, wie z. B. der Ausbruch des Vesuvs und die Vernichtung der Städte in Kampanien (79 n. Chr.), die Überflutung von Alexandria (365 n. Chr.) oder das Erdbeben von Antiochia (526 n. Chr.), in der literarischen Überlieferung und damit auch der Forschung. Es soll sogar wahre ,Katastrophenjahre' gegeben haben, wie beispielsweise das Jahr 193 v. Chr., die durch die Häufigkeit der Ereignisse bei der Bevölkerung Roms zu einem Unbehagen führten. Es ist kaum zu erwarten, dass ein Ereignis, wie es in Três Minas nachzuweisen ist, in der antiken Überlieferung je erwähnt worden wäre.

In seiner Dissertation mit dem Titel „Erdbeben. Das außergewöhnliche Normale“ beschäftigt sich G. Waldherr mit der Rezeption seismischer Aktivitäten in literarischen Quellen vom 4. Jh. v. Chr. bis zum 4. Jh. n. Chr. ${ }^{11}$ H. Sonnabend erweitert das Thema und arbeitet in seinem Werk ,Naturkatastrophen in der Antike. Wahrnehmung. Deutung. Management" die Kernthese heraus, dass die Bereitschaft zur staatlichen Katastrophenhilfe von den politischen und gesellschaftlichen Rahmenbedingungen abhängig war ${ }^{12}$.

H. Sonnabend zeigt dies anhand der großen und literarisch fassbaren Ereignisse auf. Das ist naheliegend, denn die kleinen Katastrophen waren zwar viel häufiger, sind aber literarisch kaum belegt. Die Bedeutung des Ereignisses ist allerdings für die Betroffenen weit weniger von der Größe als vielmehr von der Nähe zur Katastrophe abhängig, d. h. vom direkt erlebten Intensitätsgrad ${ }^{13}$.

Bereits in der Antike gab es unterschiedliche Erklärungsversuche für Erdbeben, Seebeben, Vulkanausbrüchen usw. Am weitesten verbreitet war die Ansicht, dass es sich um ein Eingreifen der Götter handelt, wobei auch nicht Halt davor gemacht wurde, fremden Göttern die Schuld zuzuweisen ${ }^{14}$. Darüber hinaus wurden zur Erklä-

\footnotetext{
${ }^{9}$ Nach freundlicher brieflicher Mitteilung von Univ.-Prof. Dr. K. Reicherter, Lehr- und Forschungsgebiet Neotektonik und Georisiken, RWTH Aachen University, sind in der Umgebung von Três Minas keine seismischen Aktivitäten für die römische Zeit bekannt. Allerdings können entsprechende Aktivitäten nicht ganz ausgeschlossen werden, da sich westlich an das Bergbaugebiet eine NNO-SSW verlaufende Bruchzone anschließt, in deren nördlichem Teil Thermalquellen auftreten (vgl. Abb. 1, Pedras Salgadas). Weitere Untersuchungen in der Zone könnten unsere Kenntnisse in dieser Hinsicht erweitern und eine Anpassung der hier vertretenen Interpretation erfordern.

${ }^{10}$ WALDHERR/SMOLKA 2007. S. dazu bes. die folgenden Aufsätze: J. Ganzert, Der Mars-Ultor-Tempel in Rom - ein Seismograph?; R. Schatzmann, Anmerkungen zur Annahme eines Erdbebens um die Mitte des 3. Jahrhunderts in Augusta Raurica; C. S. Sommer, Vetustate conlapsum, enemy attack or earthquake?; H. Dolenz, Ein Erdbeben in der Stadt Alt-Virunum auf dem Magdalensberg?; M. Kandler/K. Decker/G. Gangel, Archäologische Befunde von Erdbebenschäden im Raum von Carnuntum und ihre seismotektonische Interpreation; K.-G. Hinzen, Archäoseismologische Untersuchungen im Rheinland.

${ }^{11}$ WALDHERR 1997.

${ }^{12}$ SONNABEND 1999, 235.

${ }^{13}$ Olshausen 1998, 449; SonNABEnd 1999, 49; siehe auch VON Bredow 1998, 162.

14 SONNABEND 1999, 120 ff.; vgl. dazu auch VON BREDOw 1998. - Tertullian setzt sich gründlich mit der Verbindung von Naturkatastrophen, Sündhaftigkeit und Schuldzuweisung auseinander. Zur
} 
rung von Erdbeben wie auch anderen Naturkatastrophen beispielsweise Götterschlachten herangezogen ${ }^{15}$. Spätestens seit dem 7./6. Jh. v. Chr. wurde versucht, seismische Phänomene aufgrund von Naturbeobachtungen auf rationaler Basis zu erklären und damit Ursachen sowie Wirkungen aufzuzeigen bzw. Erdbeben vorauszusagen. Treffend stellt Waldherr fest: „Alle Theorien stellen Bemühungen dar, die im Zusammenhang mit einem Beben beobachteten Phänomene miteinander in Einklang zu bringen und in das jeweilige philosophische Weltbild einzupassen. [...] Die Beben sollten als naturgegeben und den Naturgesetzen folgend dargestellt und damit ohne direktes göttliches Einwirken erklärbar werden“"16. Danach riss die gelehrte Deutung von Naturkatastrophen nicht mehr $\mathrm{ab}^{17}$. Für Seneca war die wissenschaftliche Erklärung ein wichtiges Instrument, den Menschen die Furcht vor den Ereignissen zu neh$\operatorname{men}^{18}$.

Aus der Überlieferung wissen wir, dass Naturkatastrophen in der römischen Kaiserzeit ex eventu als Begleiterscheinungen von negativen politischen Entwicklungen bzw. einer Verletzung der Beziehung zwischen dem römischen Staat und den Göttern verstanden wurden und häufig als Prodigien für das Ende der Herrschaft eines Kaisers angesehen wurden ${ }^{19}$. Erwähnt seien hier beispielsweise Tiberius (14-37), Nero (54-68) und Galba (68-69) ${ }^{20}$. Eine Häufung von Naturkatastrophen während einer Regierungszeit wurde deshalb als schlechtes Omen aufgefasst. Der Gedanke war, dass die Erschütterung der Erde die Erschütterung im politisch-gesellschaftlichen Gefüge widerspiegelt ${ }^{21}$. So wurde als Grund für die vielen Naturkatastrophen während der Regierungszeit des oströmischen Kaisers Justinian (527-565) nach Prokop der schlechte Charakter des Kaisers verantwortlich gemacht. Er habe „mit heimlicher Macht und seiner dämonischen Natur“" die Unglücksfälle hervorgerufen ${ }^{22}$.

Schuldzuweisung vgl. Tertullian apol. 40,1: At e contrario illis nomen factionis accommodandum est qui in odium bonorum et proborum conspirant, qui adversum sanguinem innocentium conclamant, praetexentes sane ad odii defensionem illam quoque vanitatem, quod existiment omnis publicae cladis, omnis popularis incommodi Christianos esse in causam. Si Tiberis ascendit in moenia, si Nilus non ascendit in arva, si caelum stetit, si terra movit, si fames, si lues, statim Christianos ad leonem! adclamatur. Tantos ad unum? Grundsätzlich wurden von christlichen Autoren Naturkatastrophen als göttliche Ereignisse aufgefasst. S. SONNABEND 1999, 125.

${ }^{15}$ VON BREDOW 1998; SONNABEND 1999, 94-98.

${ }^{16}$ WALDHERR 1997, $47 \mathrm{f}$.

${ }^{17}$ Ausführlich bei WALDHERR 1997, 47-102.

${ }^{18}$ WALDHERR1997, 75 ff.; SONNABEND 1999, 179. - Grundsätzlich ist diese Erkenntnis von Seneca als psychologisch richtig einzustufen. Allerdings trifft die Angstminderung durch die Kenntnis der Ursachen für Naturkatastrophen nicht oder nur sehr bedingt zu, wie z.B. die von I. von Bredow beschriebene Erfahrung belegt (dies. 1998, 162). - Zum menschlichen Verhalten angesichts des Vesuvausbruchs vgl. WALDHERR 1997, 72 f. sowie OLSHAUSEN 1998.

${ }^{19}$ WALDHERR 1997, $157 \mathrm{f}$.

${ }^{20} \mathrm{Zu}$ Tiberius: Sueton Tib. 74: Et ante paucos quam obiret dies, turris Phari terrae motu Capreis concidit. - Zu Nero: Tacitus Ann. 16, 13: Tot facinoribus foedum annum etiam dii tempestatibus et morbis insignivere. vastata Campania turbine ventorum, qui villas arbusta fruges passim disiecit pertulitque violentiam ad vicina urbi; in qua omne mortalium genus vis pestilentiae depopulabatur, nulla caeli intemperie quae occurreret oculis. sed domus corporibus exanimis, itinera funeribus complebantur; non sexus, non aetas periculo vacua; servitia perinde et ingenua plebes raptim extingui, inter coniugum et liberorum lamenta, qui dum adsident, dum deflent, saepe eodem rogo cremabantur. equitum senatorumque interitus quamvis promisci minus flebiles erant, tamquam communi mortalitate saevitiam principis praevenirent. - $\mathrm{Zu}$ Galba: Sueton Galba 18: Vrbem quoque et deinde Palatium ingressum excepit terrae tremor et assimilis quidam mugitui sonus. - Siehe auch BARCELÓ 1998, 101.

${ }^{21}$ WALDHERR 1997, 207.

${ }^{22}$ SONNABEND 1999, 152. - Prokop beschreibt in seiner sog. Geheimgeschichte Justinian mehrfach als

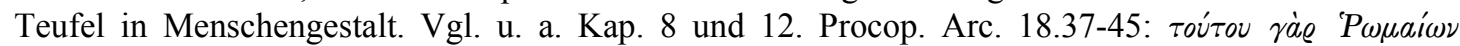

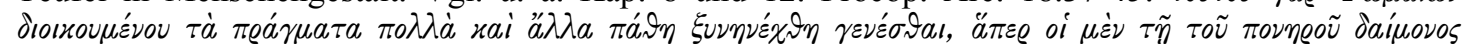


Wie erwähnt, war die Bereitschaft zur staatlichen Katastrophenhilfe grundsätzlich von den politischen und gesellschaftlichen Rahmenbedingungen abhängig, die im 1. und 2. Jh. n. Chr. als weitgehend stabil bezeichnet werden können. Es kann davon ausgegangen werden, dass praktische Hilfe als selbstverständlich angesehen wurde bzw. als Möglichkeit, sich als fürsorglicher Herrscher, als pater patriae, zu profilieren $^{23}$. Meist bestand die Unterstützung aus Geldspenden, der Streichung der Abgaben sowie der Versorgung mit qualifiziertem Fachpersonal zum Wiederaufbau ${ }^{24}$. Schließlich hatte der Herrscher nicht nur die Pflicht, sondern aus den erwähnten Gründen auch das größte Interesse daran, die Spuren einer Katastrophe so schnell wie möglich zu beseitigen.

Welchen Einfluss hatte nun diese Haltung bei einem Ereignis, wie es sich in der Galeria dos Alargamentos in Três Minas fassen lässt? Das dortige territorium metallorum war als kaiserlich-fiskalisches Eigentum aus der regionalen Verwaltung ausgegliedert und der unmittelbaren Aufsicht eines procurator metallorum unterstellt ${ }^{25}$. Dabei handelte es sich, so weit überliefert, stets um Freigelassene, die sich aus der familia Caesaris rekrutierten ${ }^{26}$. Diese Prokuratoren hatten, wie aus den erhaltenen Bronzetafeln der Bergwerksordnung von Vipasca (Aljustrel, Portugal) hervorgeht, ihre Kompetenzen vor allem in der Verwaltung sowie in der lokalen Jurisdiktion ${ }^{27}$. Damit vertraten sie qua Amt den Staat bzw. den Kaiser. H.-C. Noeske, der sich gründlich mit der Verwaltung und der Bevölkerung dakischer Bergwerke auseinandergesetzt hat, betont ausdrücklich diese Seite der kaiserlichen Prokuratoren, egal ob sie dem Freigelassenenstand oder dem Ritterstand entstammten ${ }^{28}$. Folglich ist mit Sicherheit davon auszugehen, dass der Prokurator dafür verantwortlich zeichnete, dass nach einer Katastrophe der frühere Zustand wieder hergestellt wurde.

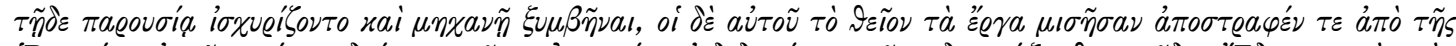

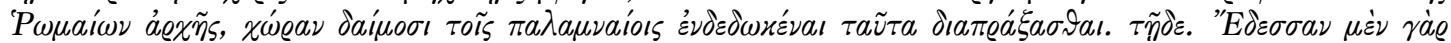

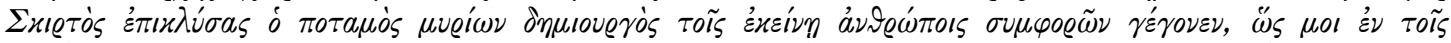

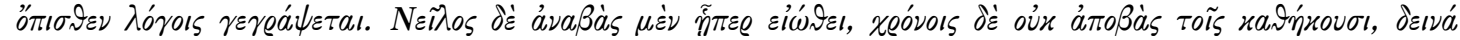

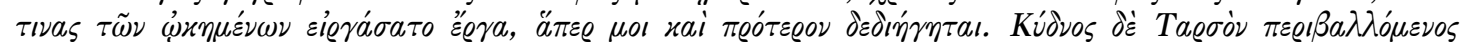

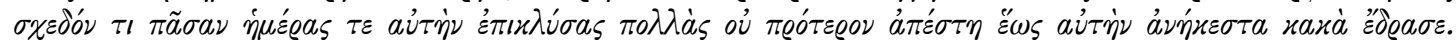

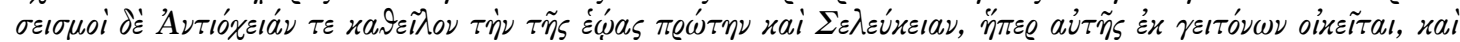

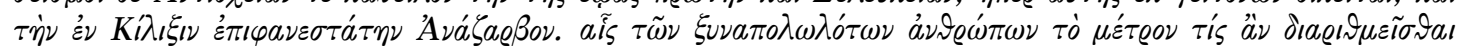

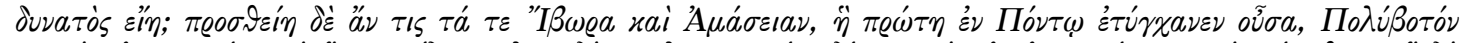

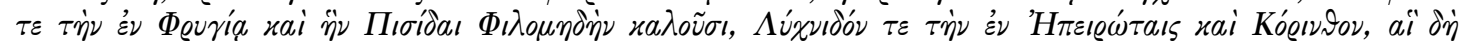

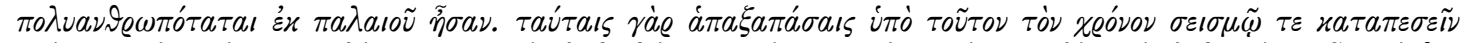

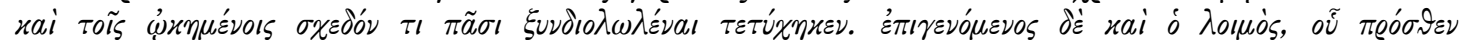

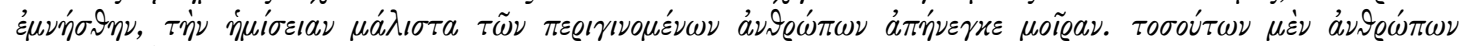

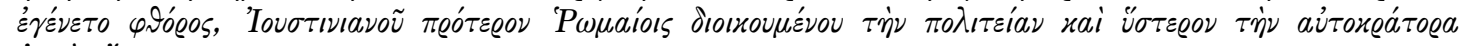

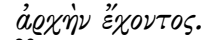

${ }^{23}$ WinTER 1998, 149. - Nach Sueton soll sich Kaiser Caligula bitter beklagt haben, dass während seiner Regierungszeit so wenige Katastrophen passiert sind. Suet. Cal. 31: Queri etiam palam de condicione temporum suorum solebat, quod nullis calamitatibus publicis insignirentur; Augusti principatum clade Variana, Tiberi ruina spectaculorum apud Fidenas memorabilem factum, suo oblivionem imminere prosperitate rerum; atque identidem exercituum caedes, famem, pestilentiam, incendia, hiatum aliquem terrae optabat. - Zur Diskussion der Mechanismen der politischen Instrumentalisierung von Naturkatastrophen, d.h. dem Umgang der Herrschenden damit, s. WALDHERR 1998, 63.

${ }^{24}$ SONNABEND 1999, 219. 245.

${ }^{25}$ WAHL 1988, 241. - HiRT 2010, 105. 119. - DOMERGUE 2008, 196 ff.

${ }^{26}$ WAHL 1988, 241.

${ }^{27}$ FlACH 1979; NoESKE 1977; HIRT 2010, 357-369. - Zur sozialen Bedeutung vgl. AlFöLdY 1984, 125.

${ }^{28}$ NOESKE 1977, $301 \mathrm{f}$.
} 
Die Bevölkerung in einem Bergwerksdistrikt umfasst sowohl wirtschaftlich wie auch sozial sehr unterschiedliche Gruppen ${ }^{29}$. H.-C. Noeske schreibt dazu: „Das soziale Gefüge der Bewohner von Alburnus maior scheint, wenn uns die erhaltenen Wachstafeln dieses Urteil gestatten, bemerkenswert differenziert gewesen zu sein. ${ }^{\text {‘30 }}$ Im Allgemeinen hat dieses Nebeneinander wohl gut funktioniert, auch wenn der personenrechtliche nicht immer dem sozialen Status entsprach. Dies war beispielsweise dann nicht der Fall, wenn ein römischer Bürger als Bergwerksarbeiter von einem Pächter mit dem Status eines Peregrinen beschäftigt wurde ${ }^{31}$. Grundsätzlich waren mit dieser Differenz von öffentlich-rechtlichem und sozialem Status Konflikte vorgeplant, vor allem wenn sich römische Bürger gegenüber einem libertus, auch wenn er der familia Caesaris entstammte, servil benehmen mussten, weil sie von diesem abhängig waren $^{32}$. Gerade in Katastrophensituationen können latente Spannungen an die Oberfläche kommen und zu sozialen Unruhen führen.

Damit verbunden ist die Frage, wer die Rettungsaktion veranlasst hat, oder ob nicht die Durchführung der Maßnahme durch einen gewissen Gemeinschaftssinn unter den Bergleuten dort unmittelbar nach der Katastrophe zum Tragen kam. Andererseits kann vermutet werden, dass es dem zuständigen procurator neben der Rettung der Bergleute ein wichtiges Anliegen war, dass die Arbeiten im Bergwerks geordnet weiterliefen und die Spuren des Ereignisses möglichst schnell beseitigt wurden.

Der Befund deutet darauf hin, dass der Vortrieb im Treppenschacht wahrscheinlich nach der vermuteten Bergungsaktion nicht mehr weitergeführt wurde. $\mathrm{Zu}$ dem gibt es keine Möglichkeit festzustellen, ob die Prospektion im Bereich der Galeria dos Alargamentos mit dem Ereignis ihren Abschluss fand. Nach der Aufgabe der Arbeiten in der Galeria dos Morcegos (Abb. 2, N), die wegen des unrentabel gewordenem Abbaus in Tagebau B (Corta da Ribeirinha) aufgelassen wurde, hat man vermutlich einen heute nicht mehr erhaltenen Altar im Eingangsbereich aufgestellt, auf den jedoch eine Einarbeitung in der Sohle hinweist ${ }^{33}$. Ob dies auch für die Galeria dos Alargamentos der Fall war, kann wegen des Schuttmaterials im Mundlochbereich nicht mehr festgestellt werden. Letztlich entzieht es sich unserer Kenntnis, ob die Rettungsaktion erfolgreich war. Es ist nicht auszuschließen, dass sich unter dem Schutt am Ende des Treppenschachts die sterblichen Überreste römischer Bergleute befinden.

\footnotetext{
${ }^{29}$ Zu Três Minas: WAHL 1988, 241. - Zur Provinz Lusitania: EDMONDSON 1987, 67 ff. - Zu den Goldbergwerken in Dakien: NOESKE 1977, passim. - In den Silberbergwerken um Cartagena (Spanien) sind römische Bürger italischer Herkunft seit dem 2. Jh. v. Chr. belegt. Vgl. EDMONDSON 1987, 61 - Siehe auch HIRT 2010, passim.

${ }^{30}$ NOESKE 1977, 339.

${ }^{31}$ NOESKE 1977, 343.

${ }^{32}$ ALFÖLDY 1984, $98 \mathrm{f}$.

${ }^{33}$ WAHL-CLERICI 2008, 54.
} 


\section{Verzeichnis der abgekürzt zitierten Literatur:}

\section{ALFÖLDY 1984}

G. Alföldy, Römische Sozialgeschichte (Wiesbaden ${ }^{3}$ 1984).

BARCELÓ 1998

P. Barceló, Die Darstellung von Naturkatastrophen in der spätantiken Literatur. In: E. Olshausen/H. Sonnabend (Hrsg.), Naturkatastrophen in der antiken Welt. Stuttgarter Kolloquium zur historischen Geographie des Altertums 6, 1996 (Stuttgart 1998) 99-104.

VON BREDOW 1998

I. von Bredow, Die mythischen Bilder der Naturkatastrophen. In: E. Olshausen/H. Sonnabend (Hrsg.), Naturkatastrophen in der antiken Welt. Stuttgarter Kolloquium zur historischen Geographie des Altertums 6, 1996. (Stuttgart 1998) 162-168.

EDMONDSON 1987

J. C. Edmondson, Two industries in Roman Lusitania. Mining and garum production. BAR Int. Series 362 (Oxford 1987).

DOMERGUE 2008

C. Domergue, Les mines antiques. La production des métaux aux époques grecque et romaine (Paris 2008).

FLACH 1979

D. Flach, Die Bergwerksordnungen von Vipasca. Chiron 9, 1979, 399-448.

HIRT 2010

A. Hirt, Imperial mines and quarries in the Roman world. Organizational aspects $27 \mathrm{BC}-\mathrm{AD} 235$ (Oxford 2010).

NOESKE 1977

H.-C. Noeske, Studien zur Verwaltung und Bevölkerung der dakischen Goldbergwerke in römischer Zeit. Bonner Jahrbücher 177, 1977, 271-416.

OLSHAUSEN 1998

E. Olshausen, Mit der Katastrophe leben. Mentalitätsgeschichtliche Studie zum Umgang von Menschen mit Naturkatastrophen am Beispiel des Vesuvausbruchs 79 n. Chr. In: E. Olshausen/H. Sonnabend (Hrsg.), Naturkatastrophen in der antiken Welt. Stuttgarter Kolloquium zur historischen Geographie des Altertums 6, 1996 (Stuttgart 1998) 448-461.

OLSHAUSEN/SONNABEND 1998

E. Olshausen/H. Sonnabend (Hrsg.), Naturkatastrophen in der antiken Welt. Stuttgarter Kolloquium zur historischen Geographie des Altertums 6, 1996 (Stuttgart 1998).

SONNABEND 1999

H. Sonnabend, Naturkatastrophen in der Antike. Wahrnehmung - Deutung Management (Stuttgart 1999).

THOMMEN 2009

L. Thommen, Umweltgeschichte der Antike (München 2009).

WAHL 1988

J. Wahl, Três Minas. Vorbericht über die archäologischen Untersuchungen im Bereich des römischen Goldbergwerks 1986/87. Madrider Mitteilungen 29, 1988, 221-244. 


\section{WAHL-CLERICI 2008}

R. Wahl-Clerici, Das römische Goldbergwerk von Três Minas. Archäologie in Deutschland 5, 2008. 54-57.

WAHL-CLERICI 2010

R. Wahl-Clerici, Untersuchungen zum Abbaufortschritt in der Corta de Covas (Tagebau A) im römischen Goldbergwerksbezirk von Três Minas (conc. Vila Pouca de Aguiar, Portugal). In: J. G. Gorges/T. Nogales Basarrate (Red.), Naissance de la Lusitanie romaine (Ier av. - Ier ap. J.-C.). VIIe Table ronde internationale sur la Lusitanie romaine (Toulouse 8-9 novembre 2007) $=$ Origen de la Lusitania romana (siglos I a.C. - I d.C.) : VII Mesa Redonda Internacional sobre la Lusitania Romana (Toulouse 8-9 novembre 2007), (Toulouse/Mérida 2010) 437-458.

WAHL-CLERICI IM DRUCK

R. Wahl-Clerici, Pilier et puits. In: Actas del Congreso Minería y Museología (Festschrift C. Domergue). León 2009. Im Druck.

WAHL-CLERICI/HELFERT/RAMMINGER 2011

R. Wahl-Clerici/M. Helfert/B. Ramminger, Mit 3D unter Tage - Römischen Bergbauingenieuren auf der Spur. Archäologie in Deutschland 2, 2011, 54-55.

WAHL-CLERICI/WIECHOWSKI IM DRUCK

R. Wahl-Clerici/A. Wiechowski, Untersuchungen zur antiken Prospektion von Erzlagerstätten mit bergbaulichen Beispielen aus dem römischen Goldbergwerksdistrikt von Três Minas / Gralheira und Campo de Jales in Nordportugal. Im Druck.

WALDHERR 1997

G. H. Waldherr, Erdbeben. Das außergewöhnliche Normale. Zur Rezeption seismischer Aktivitäten in literarischen Quellen vom 4. Jahrhundert v. Chr. bis zum 4. Jh. n. Chr. Stuttgart 1997.

WALDHERR 1998

G. H. Waldherr, Altertumswissenschaften und moderne Katastrophenforschung. In: E. Olshausen/H. Sonnabend (Hrsg.), Naturkatastrophen in der antiken Welt. Stuttgarter Kolloquium zur historischen Geographie des Altertums 6, 1996 (Stuttgart 1998) 51-64.

WALDHERR/SMOLKA 2007

G. H. Waldherr/A. Smolka (Hrsg.), Antike Erdbeben im alpinen und zirkumalpinen Raum. Befunde und Probleme in archäologischer, historischer und seismologischer Sicht (Stuttgart 2007).

WINTER 1998

E. Winter, Strukturelle Mechanismen kaiserlicher Hilfsmassnahmen nach Naturkatastrophen. In: E. Olshausen/H. Sonnabend (Hrsg.), Naturkatastrophen in der antiken Welt. Stuttgarter Kolloquium zur historischen Geographie des Altertums 6, 1996 (Stuttgart 1998) 147-155. 
Frankfurter elektronische Rundschau zur Altertumskunde 17 (2012)

Abbildungen:

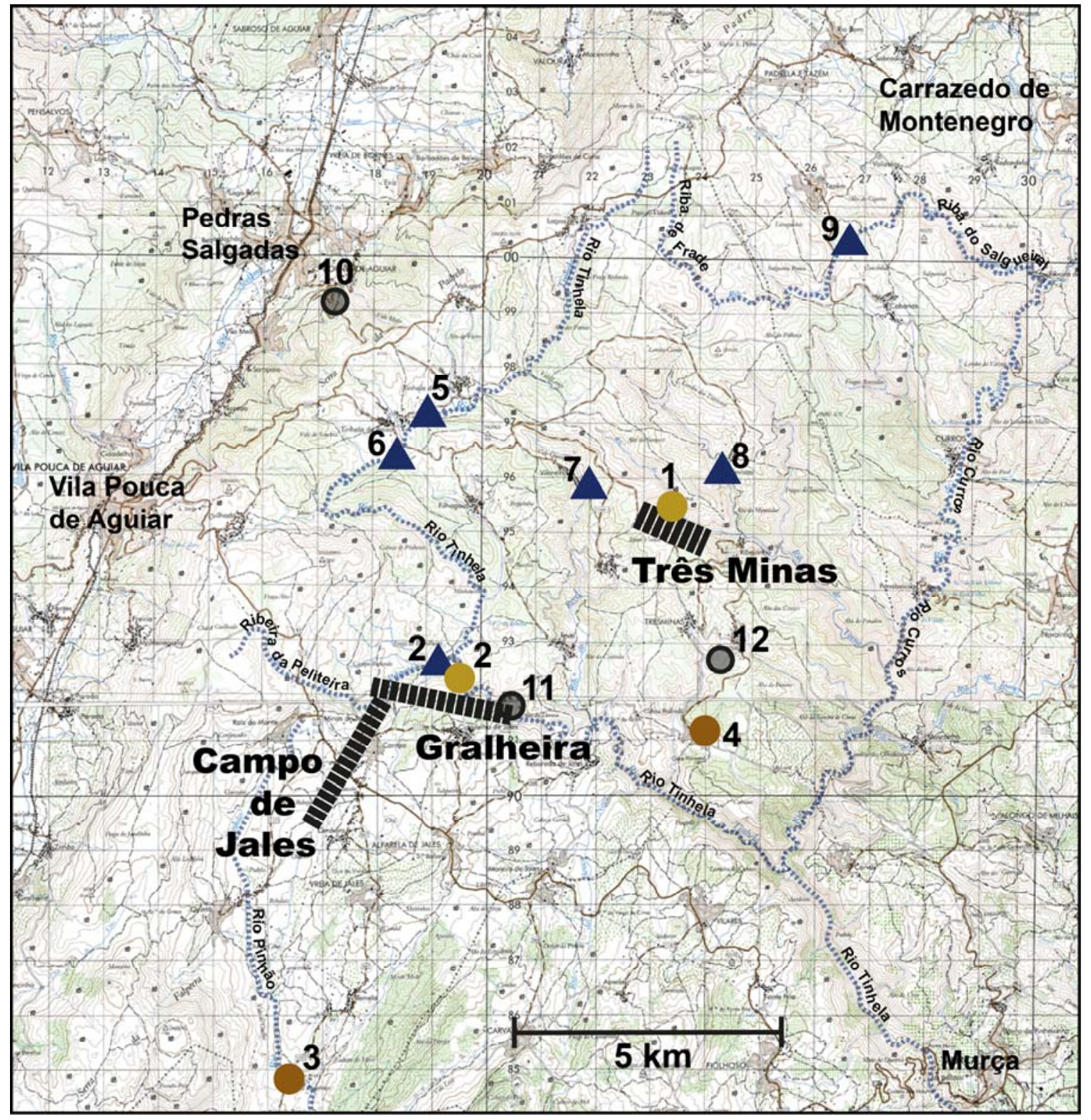

Abb. 1. Übersicht über die Bergwerkszone von Três Minas, Gralheira und Campo de Jales

1 Waschanlagen

2 Forno dos Mouros mit dazugehörigem Staudamm

3 Römische Brücke von Barrela über den Rio Pinhão

4 Römischer Landwirtschaftsbetrieb von Vales

5 Erddamm von Outeiro

Kleine Schrift: moderne Orte bzw. Flüsse

(Vorlage Instituto Geográfico do Exército. Carta Militar de Portugal. 1:50'000. Folhas 6-II/10.1. Bearbeitung Wahl und Wahl-Clerici)
6 Erddamm von Tinhela de Baixo

7 Staumauer von Vilarelho

8 Stauwehr von Sevivas

9 Erddamm von Tázem

10 Prähistorisches Castro von Bornes de Aguiar

11 Prähistorisches Castro von Cidadelha

12 Westgotische Gräber von Granja 


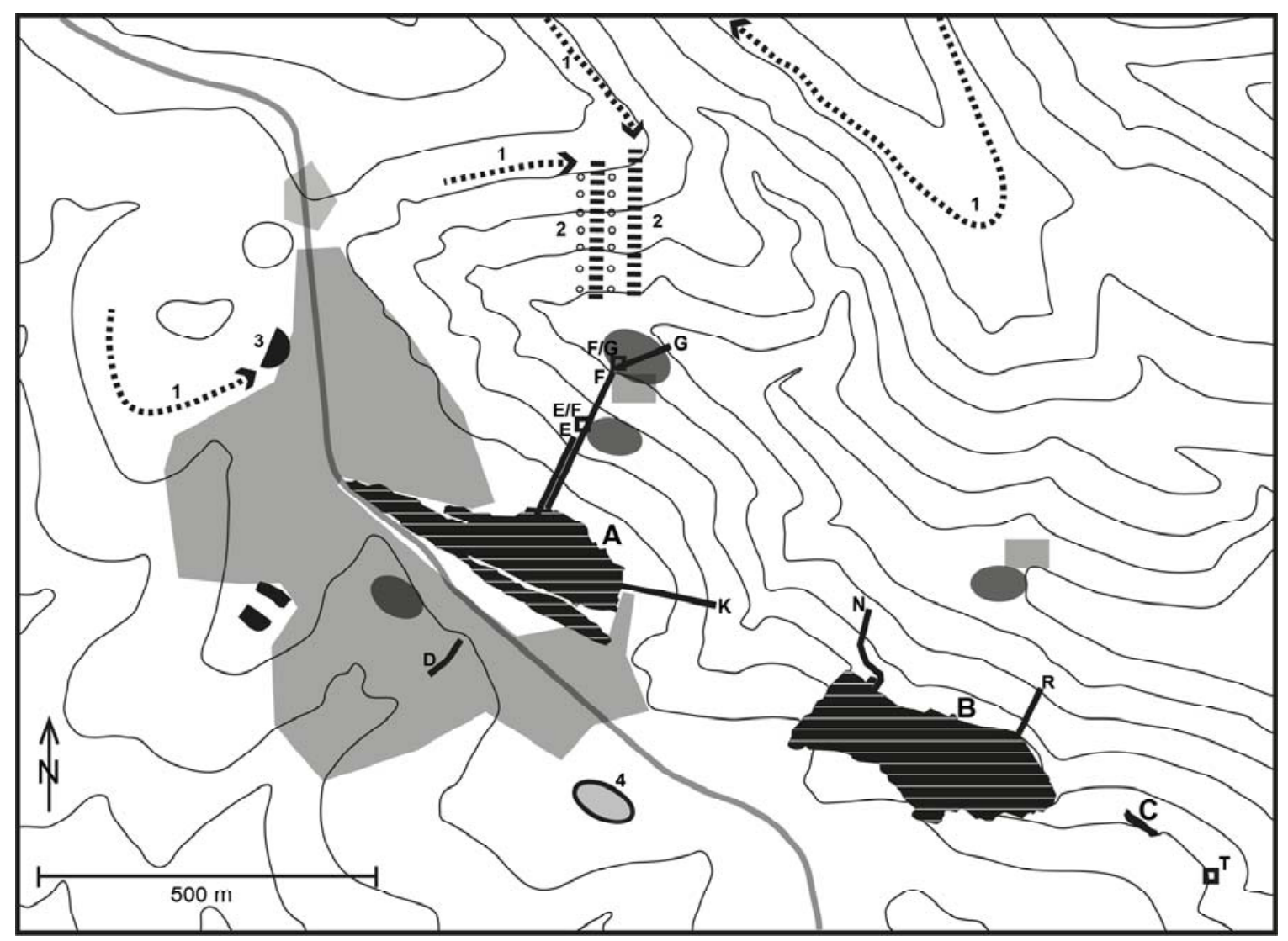

Abb. 2 Três Minas. Kernzone des Bergbaus
A Corta de Covas (Tagebau A)
B Corta da Ribeirinha ( Tagebau B)
C Lagoinhos (Abbaustelle C)
D Galeria Esteves Pinto
E Galeria Jürgen Wahl
E/F Schacht zwischen Galeria Jürgen Wahl und Galeria do Pilar
F Galeria do Pilar
F/G Schacht Galeria do Pilar und Galeria do Texugo

G Galeria do Texugo

K Galeria dos Alargamentos

N Galeria dos Morcegos

R Galeria do Buraco Seco

$\mathrm{T}$ Prospektionsbaue

1 Aquädukte mit Verlaufsrichtung

2 Waschanlagen, westliche Reihe mit Absetzbecken

3 Wasserabsetzbecken in der Siedlung

4 Amphitheater

Hellgrau: Siedlungszonen, dunkelgrau: Aufbereitungszonen

(Vorlage Wahl. Bearbeitung Helfert, Wahl-Clerici) 
Frankfurter elektronische Rundschau zur Altertumskunde 17 (2012)

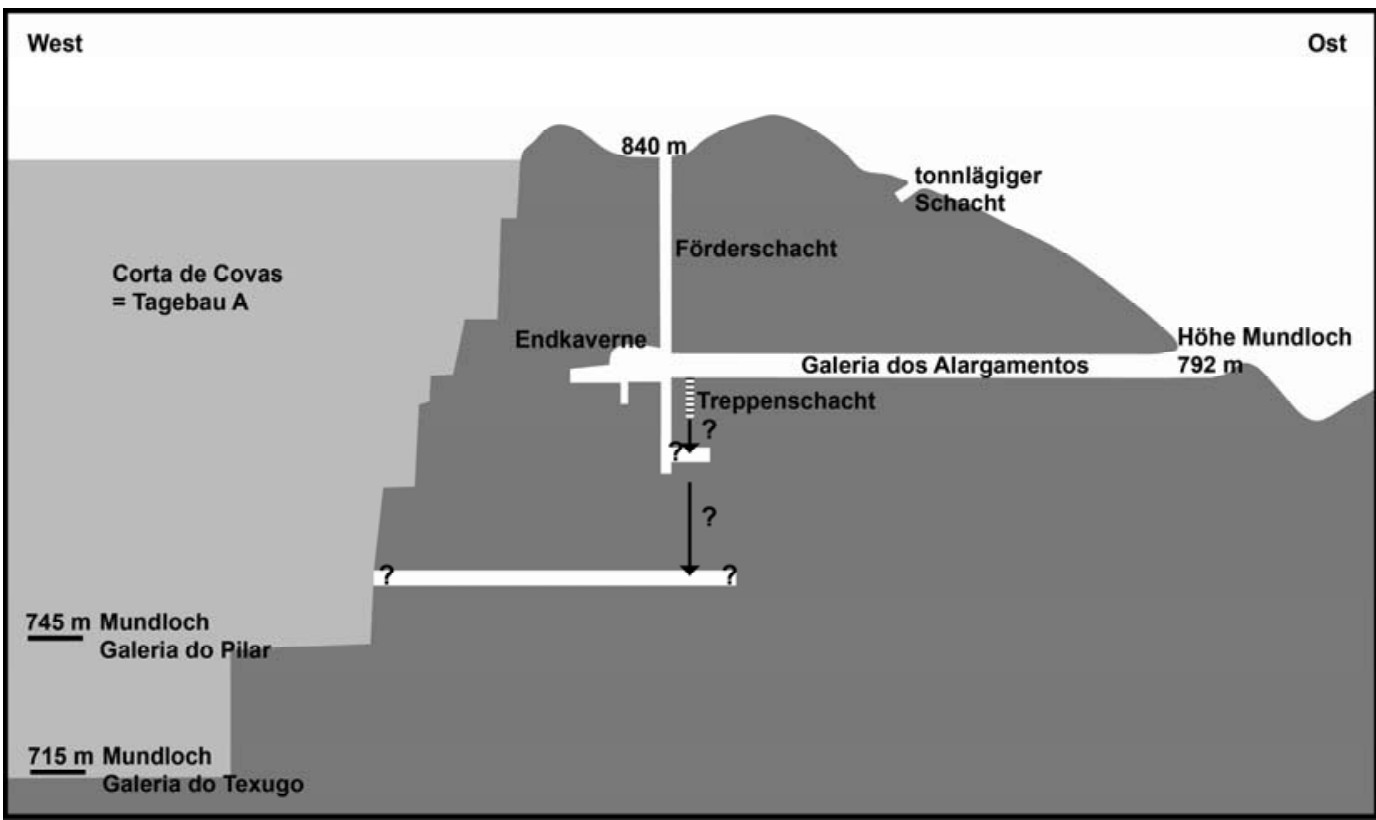

Abb. 3 Três Minas. West-Ost orientierter Querschnitt durch den Hang, der östlich an die Corta de Covas (Tagebau A) anschließt (stark schematisiert). Die Abbildung zeigt einen älteren als den heute sichtbaren Zustand. Die beiden in Abb. 4 in der Ostwand der Corta de Covas erkennbaren Schächte wurden erst später durch einen Bergsturz freigelegt. Die gestrichelte Linie markiert den heutigen Verlauf der freiliegenden Oberfläche der Corta de Covas. (Vorlage und Ausführung Wahl-Clerici)

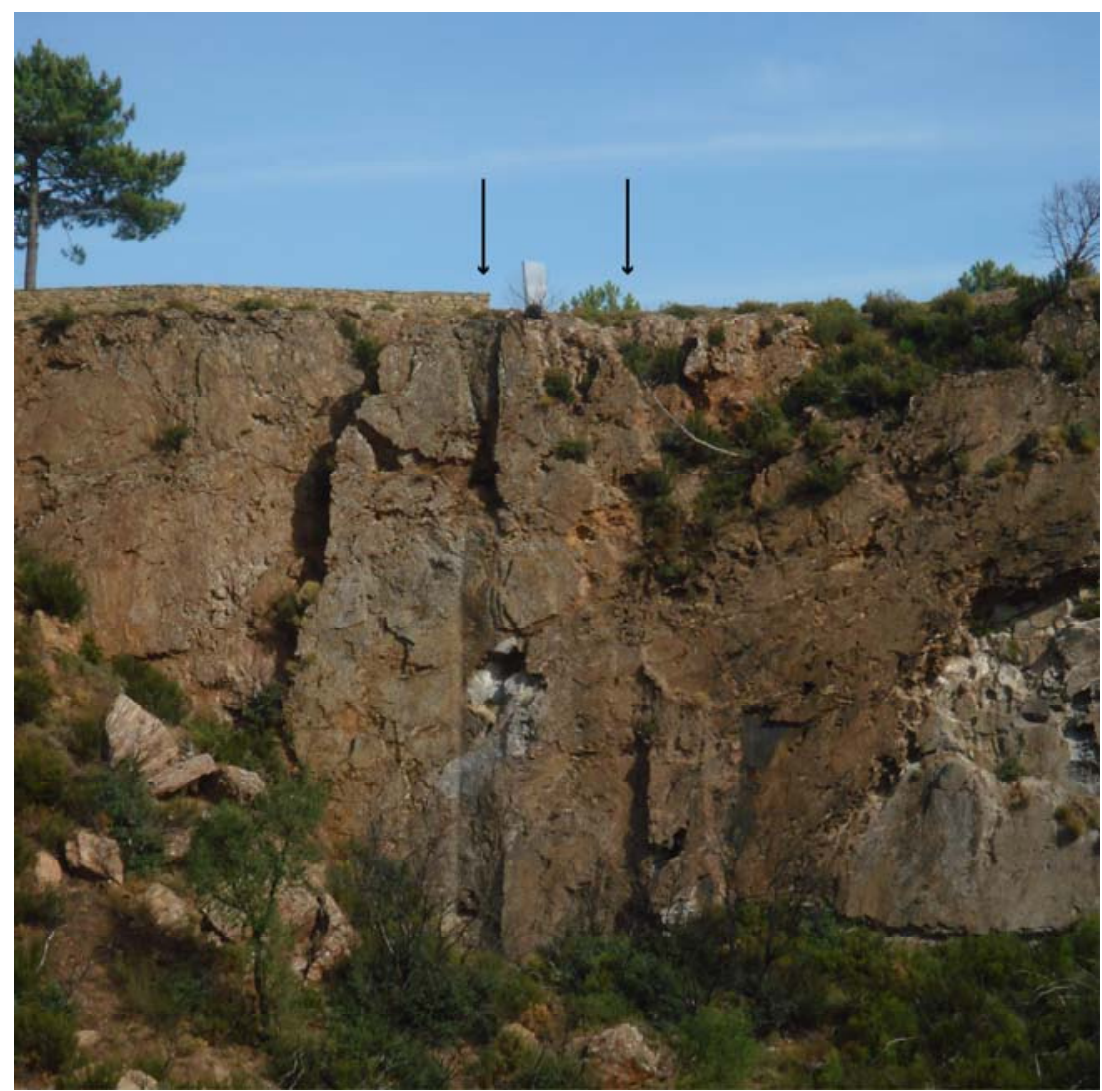

Abb. 4 Três Minas. Ostwand der Corta de Covas mit Förder- (links) und Steigschacht (rechts). Von Nordwest. (Foto Wahl-Clerici) 


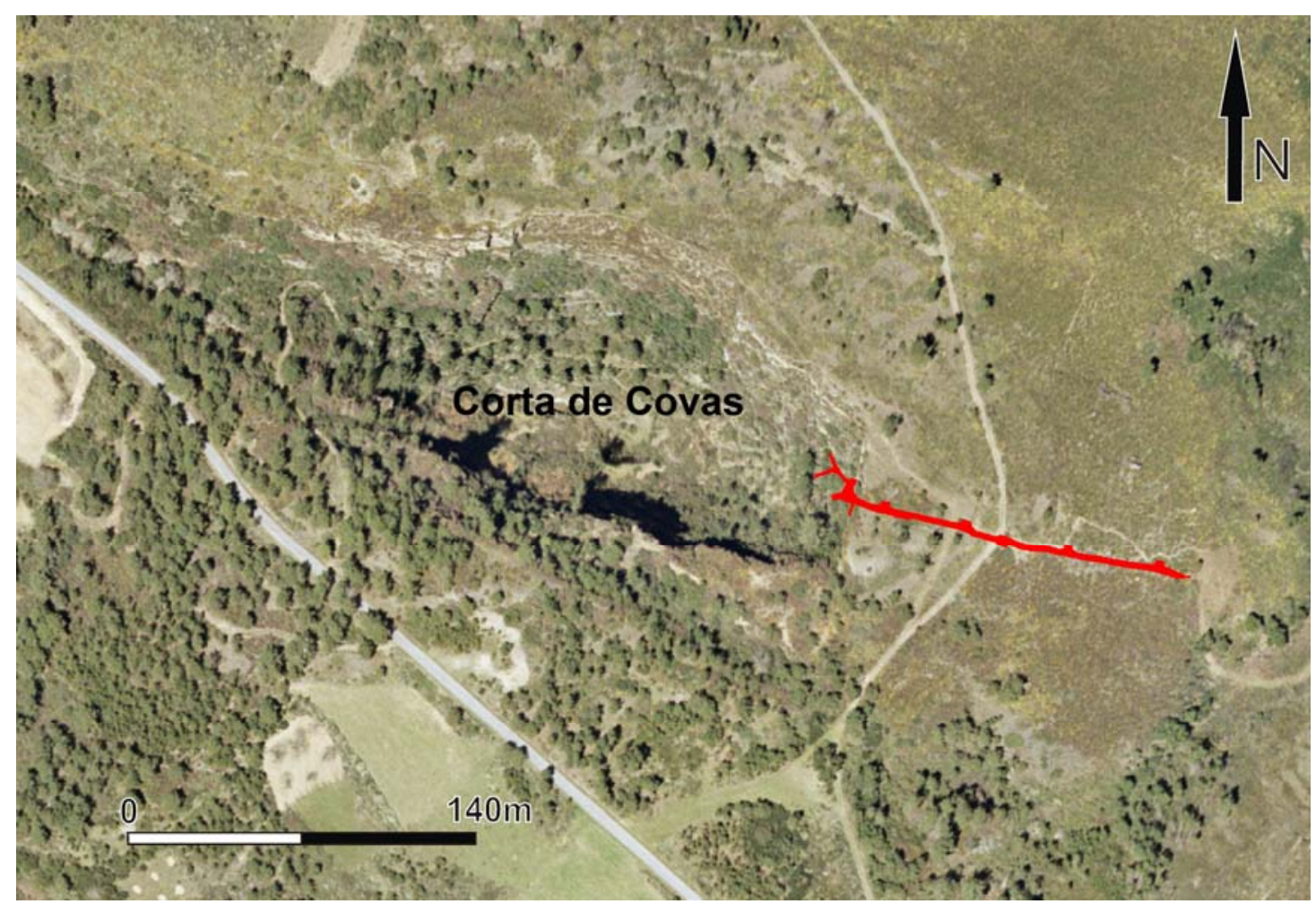

Abb. 5 Três Minas. Corta de Covas. Rot: Der unterirdische Verlauf der Galeria dos Alargamentos. Der Treppenschacht weicht von der Galerie nach Süden ab.

(Ortofotomapa 1:5000 Câmara Municipal Vila Pouca de Aguiar. 3D-Laserscanaufnahme und Bearbeitung Helfert)

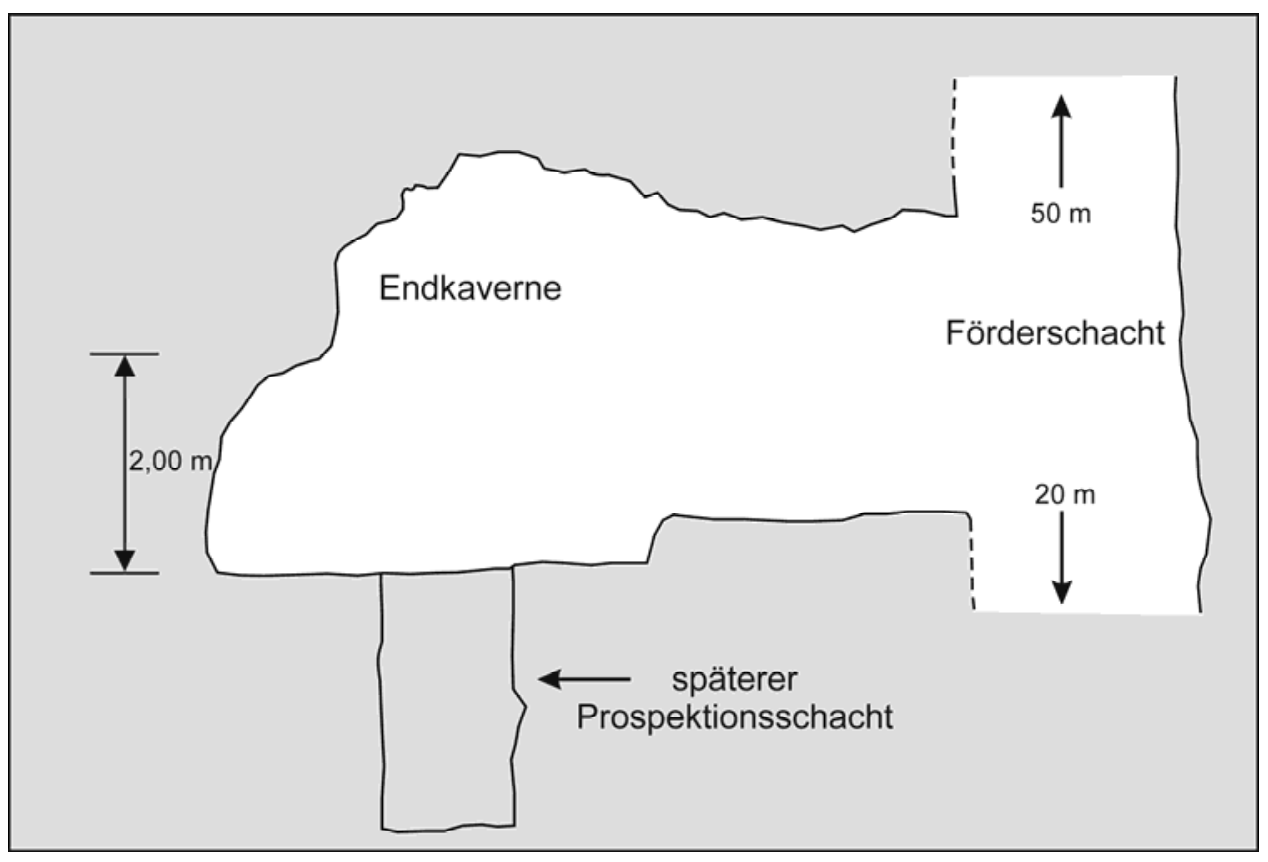

Abb. 6 Três Minas. Galeria dos Alargamentos. Endkaverne von Osten.

(Querschnitt auf Grundlage der 3D-Laserscan-Vermessung. Bearbeitung Helfert) 
Frankfurter elektronische Rundschau zur Altertumskunde 17 (2012)

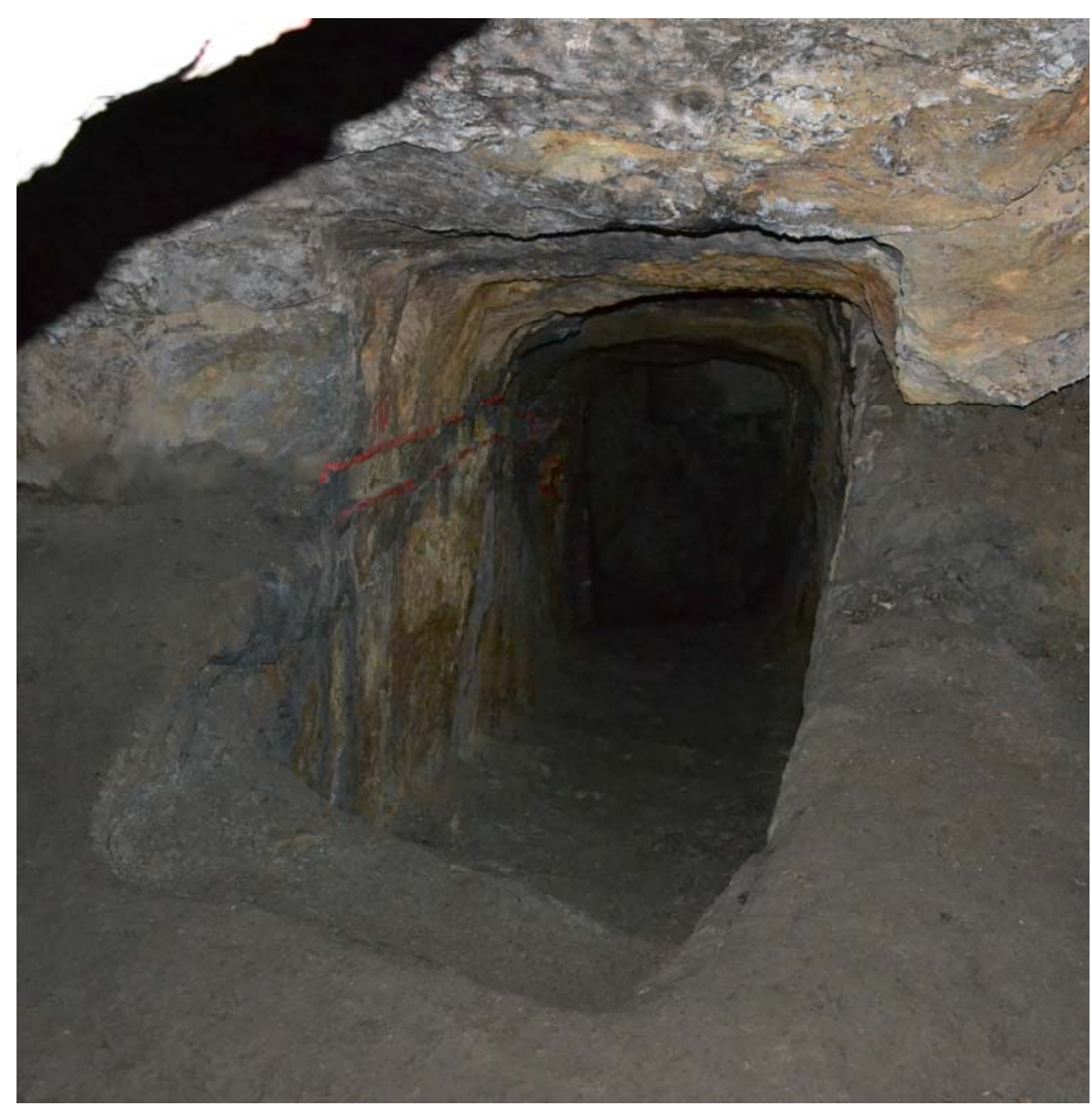

Abb. 7 Três Minas. Galeria dos Alargamentos. Treppenschacht im südlichen Stoß.

Er weist eine leichte Krümmung nach Osten auf. Die ersten Treppenstufen durchstossen die Sohle der Galerie. Im Hintergrund sind der abgesenkte Felsblock und der verkeilte Felsblock sichtbar (= Position B in Abb. 8. 9). Von Norden. (Foto Helfert)

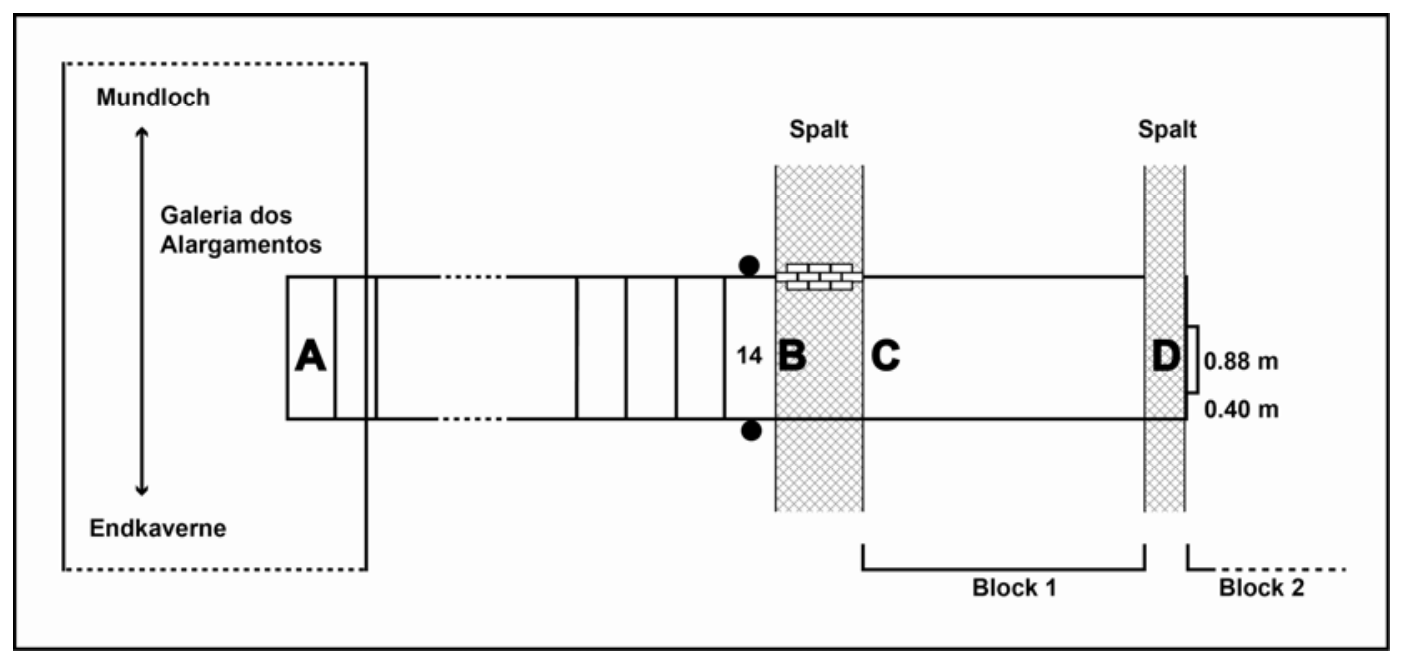

Abb. 8 Três Minas. Galeria dos Alargamentos. Treppenschacht. Stark schematisierter Grundriss. Kariert: Spalten. Schwarz gefüllte Kreise: Einarbeitungen zur Verkeilung eins Balkens. (Vorlage und Ausführung Wahl-Clerici) 
Frankfurter elektronische Rundschau zur Altertumskunde 17 (2012)

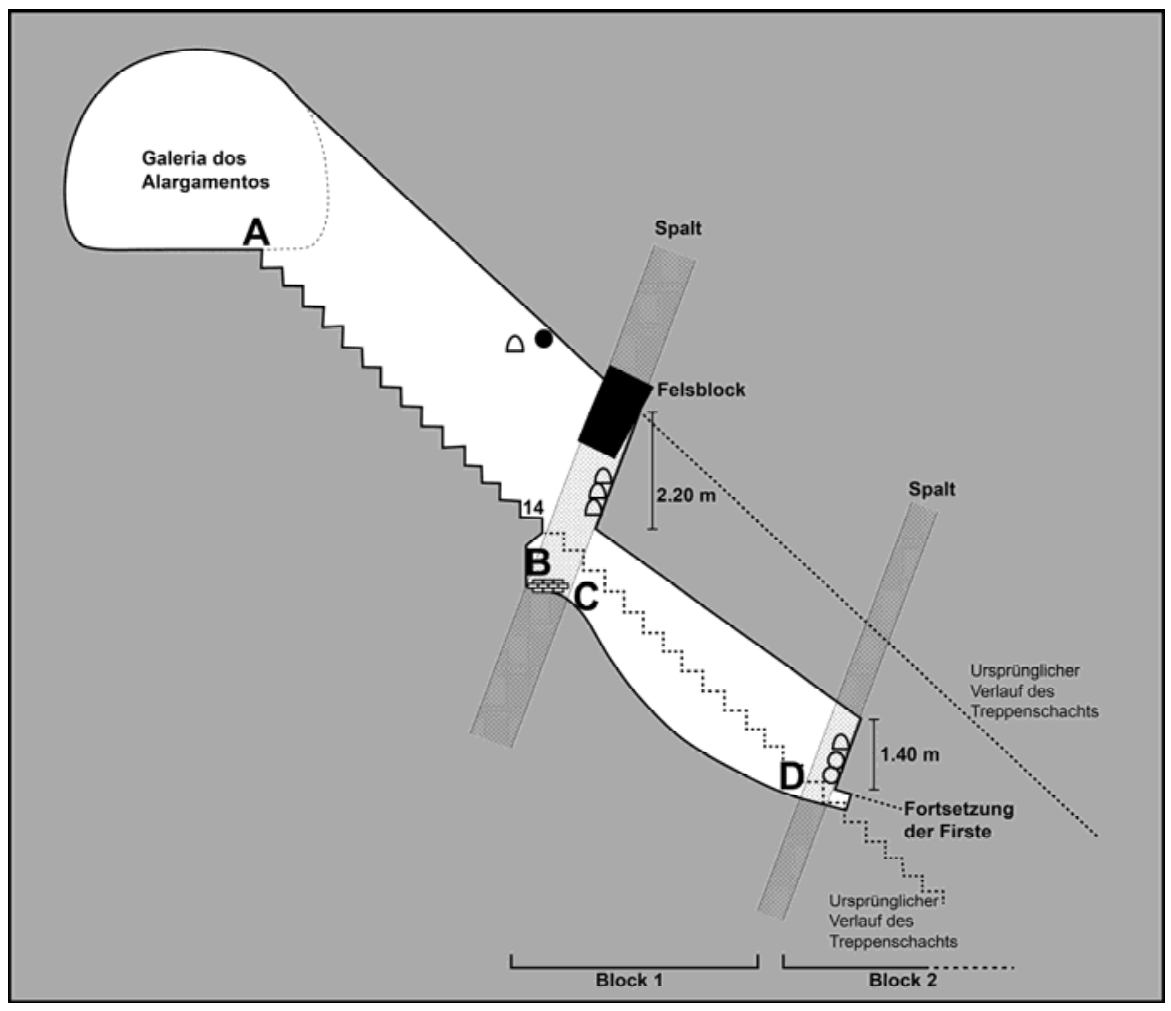

Abb. 9 Três Minas. Galeria dos Alargamentos. Treppenschacht.

Stark schematisierter Seitenriss. Schwarz gefüllter Kreis: Einarbeitung zur Verkeilung eines Balkens. Kreise: runde Einarbeitungen. Halbovale: Lampennischen.

(Vorlage Wahl-Clerici, Helfert. Ausführung Wahl-Clerici)

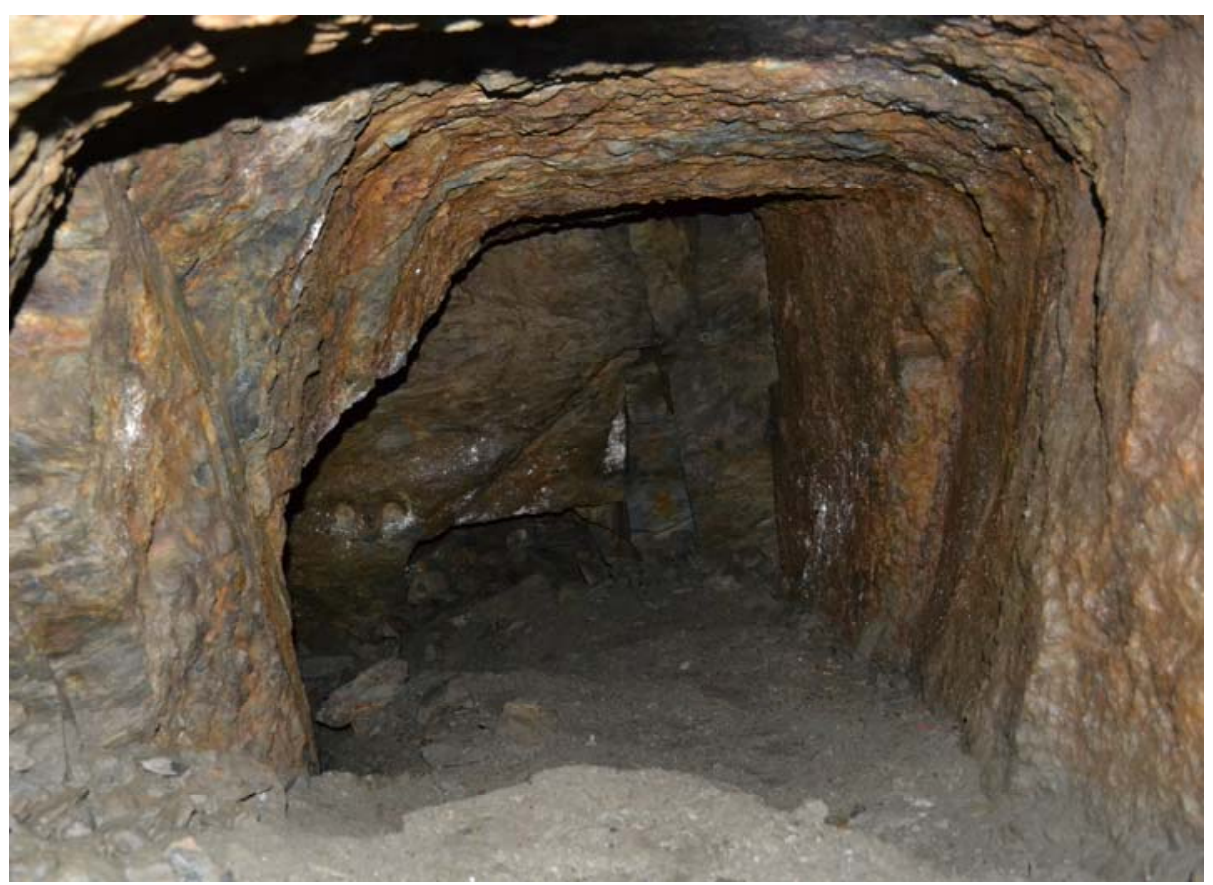

Abb. 10 Três Minas. Galeria dos Alargamentos. Treppenschacht.

Situation zwischen oberem und unterem Spalt. Blick von C nach D (s. Abb. 8. 9). Firste und westlicher Stoß sind vollständig, östlicher Stoß ist teilweise abgebrochen. Bei Position D ist die abgesenkte Fortsetzung des Treppenschachts als Profil in der Felswand und östlich daran anschliessend sind die beiden runden Einarbeitungen erkennbar. (Foto Helfert) 
Frankfurter elektronische Rundschau zur Altertumskunde 17 (2012)

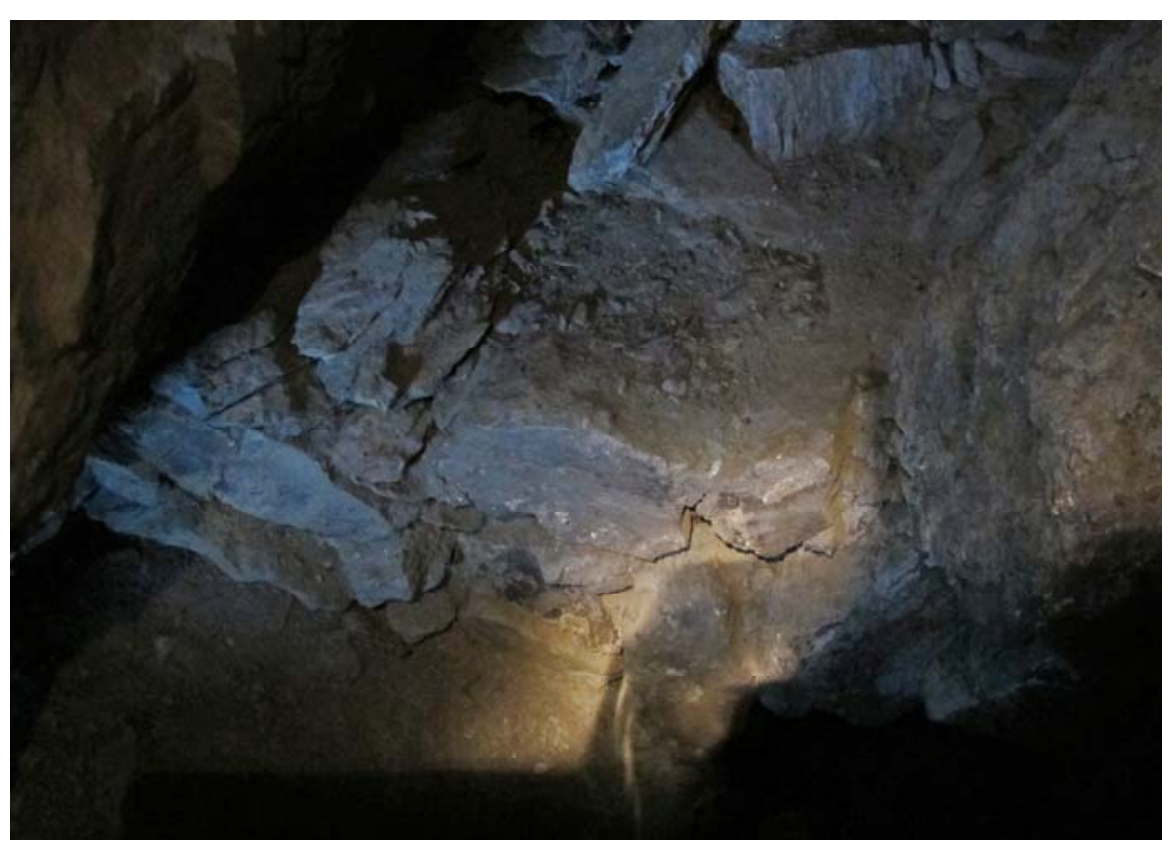

Abb. 11 Três Minas. Galeria dos Alargamentos. Treppenschacht. Position B-C (s. Abb. 8. 9). Rest der Mauer, die zur Sicherung vor seitlichen Schutteinbrüchen im oberen Spalt gebaut wurde. Von Südwesten. (Foto Wahl-Clerici)

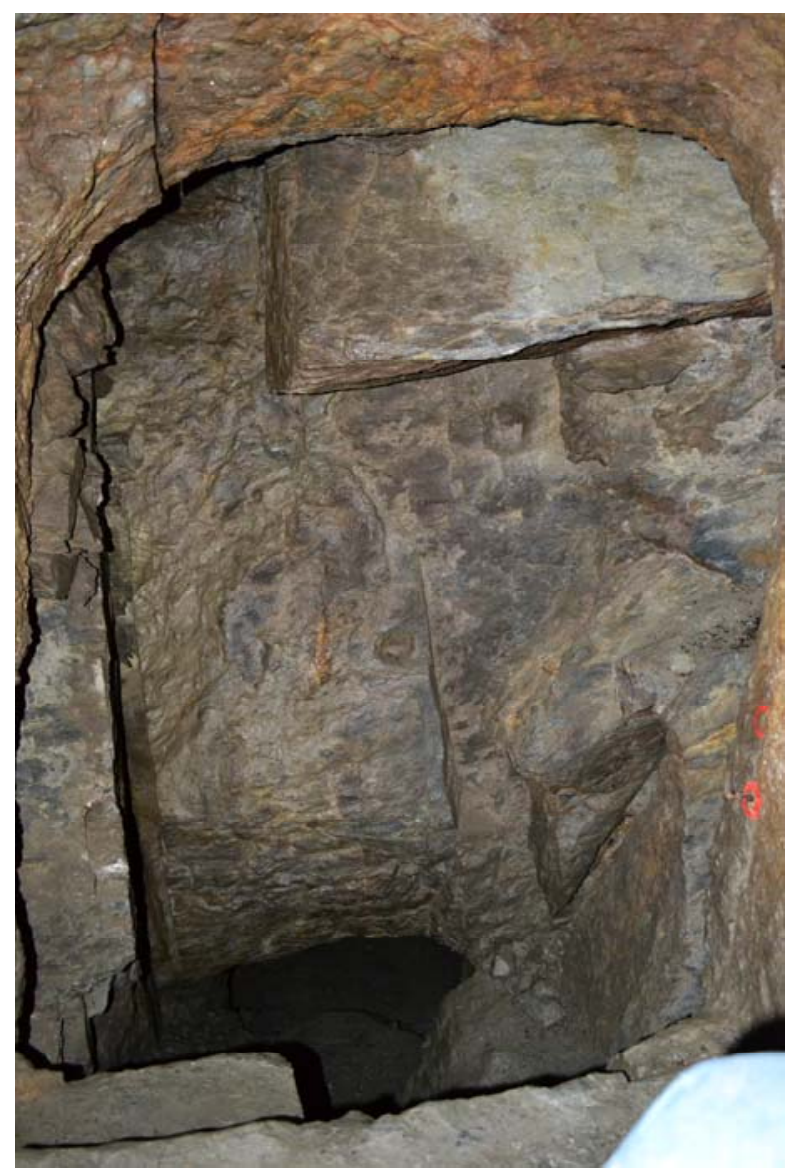

Abb. 12 Três Minas. Galeria dos Alargamentos. Treppenschacht. Pos. B und C (s. Abb. 8. 9) mit dem in römischer Zeit nicht mehr erweiterten Durchlass. Erkennbar ist die hälftig erhaltene Stufe 14 sowie der abgesenkte Felsblock mit den drei Einarbeitungen und der im Spalt verkeilte Block. Von Norden. (Foto Helfert) 
Frankfurter elektronische Rundschau zur Altertumskunde 17 (2012)

\section{Adressen der Autoren:}

Dr. M. Helfert, Institut für Archäologische Wissenschaften, Abt. II, der GoetheUniversität zu Frankfurt am Main, Grüneburgplatz 1, 60323 Frankfurt/Main.

M.Helfert@em.uni-frankfurt.de

Lic. phil. Regula Wahl-Clerici, Projecto - Três Minas - Projekt, Rainweg 3, CH-8810 Horgen

regulawahl@gmail.com

Dr. Annemarie Wiechowski, Wüllnerstr. 2, 52062 Aachen

wiechowski@,rwth-aachen.de 